\title{
Direction reversal of a rotating wave in Taylor-Couette flow
}

\author{
J. ABSHAGEN 1 M. HEISE ${ }^{1}, \mathrm{CH}$. HOFFMA N N \\ AND G. PFISTER ${ }^{1}$ \\ ${ }^{1}$ Institute of Experimental and Applied Physics, University of Kiel, 24098 Kiel, Germany \\ ${ }^{2}$ Institute for Theoretical Physics, Saarland University, 66123 Saarbrücken, Germany
}

(Received 14 February 2008 and in revised form 2 May 2008)

In Taylor-Couette systems, waves, e.g. spirals and wavy vortex flow, typically rotate in the same direction as the azimuthal mean flow of the basic flow which is mainly determined by the rotation of the inner cylinder. In a combined experimental and numerical study we analysed a rotating wave of a one-vortex state in small-aspectratio Taylor-Couette flow which propagates either progradely or retrogradely in the inertial (laboratory) frame, i.e. in the same or opposite direction as the inner cylinder. The direction reversal from prograde to retrograde can occur at a distinct parameter value where the propagation speed vanishes. Owing to small imperfections of the rotational invariance, the curves of vanishing rotation speed can broaden to ribbons caused by coupling between the end plates and the rotating wave. The bifurcation event underlying the direction reversal is of higher codimension and is unfolded experimentally by three control parameters, i.e. the Reynolds number, the aspect ratio, and the rotation rate of the end plates.

\section{Introduction}

Rotating waves are a generic flow state that appears in many rotating fluid flow systems, such as in rotating shear flows and rotating convection (see e.g. Swinney \& Gollub 1981; Mullin 1994; Egbers \& Pfister 2000), with widespread relevance to applications, e.g. in atmospheric flows (Gill 1982). Often, rotating waves appear from a hydrodynamic instability of rotationally invariant basic flow, i.e. the flow undergoes a Hopf bifurcation breaking the $S O(2)$ symmetry. Owing to the rotational invariance of the underlying fluid dynamical system, rotating waves appear steady in a certain co-rotating frame and are thus also referred to as 'relative equilibria', as discussed, e.g. in Golubitsky, Stewart \& Schaeffer (1988).

In flows with an additional reflection symmetry, e.g. the van Kármán flow between exactly counter-rotating disks (Nore, Tuckerman, Daube \& Xin 2003), the resulting $O(2)$ symmetry gives rise to two oppositely rotating waves to appear from a symmetry-breaking Hopf bifurcation (Golubitsky et al. 1988). An additionally imposed (rotational) mean flow breaks the reflection symmetry and the appearance of the prograde rotating wave is enhanced; but the retrograde wave also results naturally from a Hopf bifurcation with broken $O(2)$ symmetry (van Gils \& Mallet-Paret 1986; Crawford \& Knobloch 1988; Knobloch 1996; see also Abshagen et al. 2007 for the case of propagating instead of rotating waves).

Many rotating flows have only $S O(2)$ instead of broken $O(2)$ symmetry and thus, a Hopf bifurcation breaking rotational invariance leads only to a single rotating wave. 
Such states arise from cylindrical flows between rotating disks (Serre, Crespo del Arco \& Bontoux 2001; Marques, Lopez \& Shen 2002; and Moisy et al. 2004) or rotating spheres (Marcus \& Tuckerman 1987) (see also Egbers \& Pfister 2000). One of the most famous rotating waves in a shear flow is the so-called wavy vortex flow, that occurs in the viscous fluid in the gap between two concentric rotating cylinders, i.e. in the Taylor-Couette system (Coles 1965; Mullin \& Benjamin 1980; Jones 1981; Ahlers, Cannell \& Dominguez Lerma 1983; Marcus 1984; Mullin 1985; Nagata 1988; Edwards, Beane \& Varme 1991). Other types of rotating waves in this flow system, differing in azimuthal wave speed and number as well as in axial symmetry, such as axially sub-harmonic modes, are found by Jones (1985) and Iooss (1985). These modes have been investigated in detail (e.g. DiPrima \& Swinney 1981; Andereck, Lui \& Swinney 1986; Gerdts et al. 1994; Egbers \& Pfister 2000).

Common to all types of rotating waves found so far in Taylor-Couette flow is that they propagate in the prograde direction in the inertial (laboratory) frame, but in the retrograde direction in the frame rotating with the inner cylinder. Retrograde waves in the rotating frame appear also among others in rotating convection (Zhong, Ecke \& Steinberg 1991; Goldstein et al. 1993; Marques et al. 2007) and in atmospheric flow where large planetary waves can propagate westwards in the mid latitudes while the mean flow is eastwards (Gill 1982). However, these examples of retrograde waves are all rotating progradely in the inertial frame.

In small-aspect-ratio Taylor-Couette flow, Pfister, Schulz \& Lensch (1991) discovered experimentally a rotating-wave regime that is separated by a finite control parameter interval of zero oscillation frequency. Marques \& Lopez (2006) have confirmed numerically the experimental results from Pfister et al. (1991) for a single aspect ratio including the azimuthal wavenumber $m=2$ and a slightly subcritical Hopf bifurcation.

In our experimental study, we expand the control parameter space by an additional control parameter, namely the rotation frequency of the end plates, and additionally performed numerical simulations of the Navier-Stokes equations in order to show that the rotating wave undergoes a direction reversal between prograde and retrograde propagation in the inertial (laboratory) frame, which is organized by a bifurcation of higher codimension. In particular, a strong influence of (unavoidable) experimental imperfections of the rotational invariance on this bifurcation in some parameter regimes seems to be responsible for the appearance of finite intervals (ribbons) with zero frequency.

\section{Experimental set-up and numerical methods}

The measurements were performed in two different Taylor-Couette set-ups - both with a rotating inner and a non-rotating outer cylinder. The inner cylinder is machined from stainless steel having a radius of $r_{i}=(12.50 \pm 0.01) \mathrm{mm}$, while the outer one is made from optically polished glass with a radius of $r_{o}=(25.00 \pm 0.01) \mathrm{mm}$. At top and bottom, the fluid is confined by end plates which can rotate synchronously and independently from the inner cylinder with a frequency $f_{\text {end }}$ in one of the two apparatuses. The tilt of each rotating end plate is better that $0.03 \mathrm{~mm}$ at the outer diameter. In the other set-up, the end plates are held fixed in the laboratory frame. The distance between these plates defines the axial height $L$ of the flow and is adjustable within an accuracy of $0.01 \mathrm{~mm}$ up to a maximal height of $300.00 \mathrm{~mm}$. In order to investigate the influence of the above mentioned imperfections systematically, 

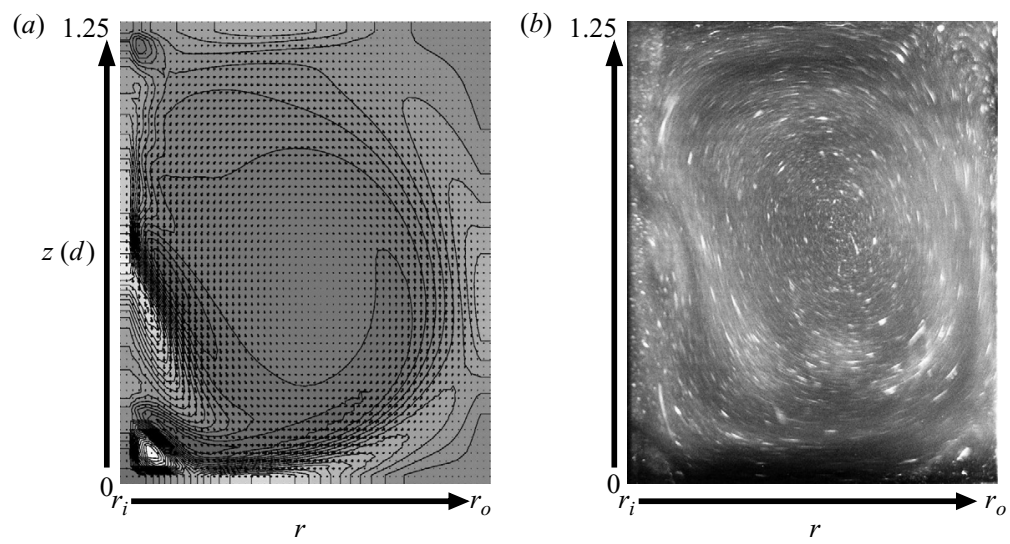

FIGURE 1. Snapshot of the velocity field over the $(r, z)$-plane depicting oscillatory single-cell flow at $\Gamma=1.25$ and $f_{\text {end }}=0$ from $(a)$ numerical simulation (contours represent $v_{\varphi}=$ const) for $R e_{i}=1190$ and $(b)$ photograph of a laser light sheet for $R e_{i}=1200$.

an additional well-defined tilt of the upper end plate can be applied in the set-up with non-rotating end plates.

Geometric parameters of the Taylor-Couette flow are the aspect ratio $\Gamma=L / d$, with the gap width $d=r_{o}-r_{i}$, and the radius ratio $\eta=r_{i} / r_{o}$ which is held fixed at $\eta=0.5$ for all measurements. Within the gap between the two concentric cylinders, silicone oil with two different kinematic viscosities $v=(5.7 \pm 0.1) \mathrm{cS}$ and $(v=10.9 \pm 0.1)$ $\mathrm{cS}$ is used as the working fluid. The inner cylinder Reynolds number $R e_{i}=2 \pi f_{i} r_{i} d / v$ with $f_{i}$ denoting the rotation frequency of the inner cylinder, serves as one control parameter. The rotation frequency of the end plates $f_{\text {end }}$ (given in units of diffusion time $\tau=d^{2} / \nu$ ) serves as a further control parameter. With a PLL-control, an accuracy of $\Delta f / f=10^{-4}$ in the short-term and $\Delta f / f=10^{-7}$ in the long-term average is achieved for the rotating frequency of both the inner cylinder and the end plates. We use laser-Doppler velocimetry (LDV) for contact-free measurements of the axial component of the local flow velocity.

The flow is described by the Navier-Stokes equations for incompressible fluids. Using cylindrical coordinates, we decompose the velocity field $\boldsymbol{u}=v_{r} \boldsymbol{e}_{r}+v_{\varphi} \boldsymbol{e}_{\varphi}+v_{z} \boldsymbol{e}_{z}$ into a radial component $v_{r}$, an azimuthal one $v_{\varphi}$, and an axial one $v_{z}$. For numerical calculations, we used a combined Galerkin and finite-differences code in the axial and the radial direction considering homogeneous grids with discretization lengths $\Delta r=\Delta z=0.025 d$ which have been shown to be more accurate than non-homogeneous grids. In the azimuthal direction, eight Fourier modes are used. At the axial end plates, finite boundary conditions were imposed by rigid rotating lids. Staggered grids are chosen in order to avoid discontinuities in the corners and to minimize phase errors. Time steps are $\Delta t<1 / 70000 \tau$, where $\tau=d^{2} / v$ is the diffusion time (see Hoffmann, Lücke \& Pinter 2005 for further details).

\section{Results}

A snapshot of the velocity field in the $(r, z)$-plane taken from a time-periodic single cell flow is depicted in figure 1. Both the numerically calculated flow field (figure $1 a$ ) and a photograph from a laser light sheet (figure $1 b$ ) display a large vortex with an outward directed flow close to the lower and an inward directed one close to the upper 
(a)

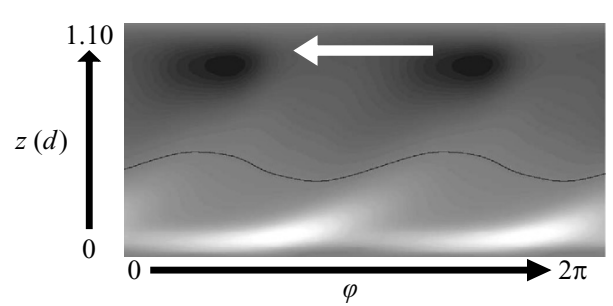

(b)

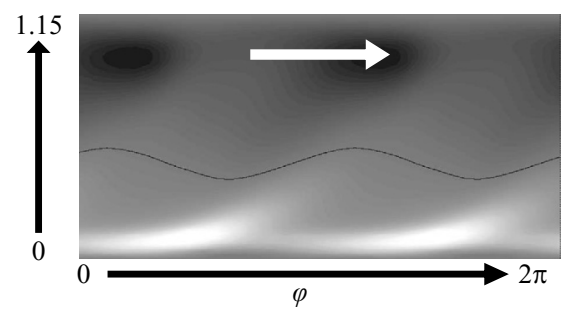

FIGURE 2. Numerically calculated radial velocity field $v_{r}$ (greycode: inflow $=$ black, outflow $=$ white) on a cylindrical surface with $r=r_{i}+0.25 d R e_{i}=800$ and $f_{\text {end }}=-4$ for $(a) \Gamma=1.10$ and $(b) \Gamma=1.15$. The white arrows indicate the propagation direction of the whole structure.

end plate. Thus, the flow is directed downwards in the vicinity of the inner cylinder. Here, a smaller, counter-rotating vortex can be seen in figure 1 near the mid-height of the system. The structure rotates as a whole, e.g. showing a time-periodic cycle when viewed from a $\varphi=$ const plane: the small vortex first moves downwards from top to bottom, then seems to disappear close to the lower lid, and reappears close to the upper end. The apparent axial propagation of the small vortex in a $\varphi=$ const plane results from the three-dimensional flow structure which has the form of a rotating wave with azimuthal wavenumber $m=2$ (Pfister et al. 1991; Marques \& Lopez 2006) and is also confirmed in our numerical simulations. Note, that a similar time-periodic one-cell state exists for comparable control parameter values on the other asymmetric branch (Pfister et al. 1991).

Visualizations of the time-periodic one-cell flow in another parameter regime $\left(R e_{i}=800\right.$ and $\left.f_{\text {end }}=-4\right)$ for two different aspect ratios are given in figure 2. Each plot represents the numerically calculated, radial velocity field $v_{r}$ on a $(z, \varphi)$-cylinder surface at $r=r_{i}+0.25 d$.

The structures of both flow states presented in figure 2 are almost identical, though they differ in $\Gamma$. The rotating wave is anharmonic, particularly close to the inner cylinder which gives rise to the apparent propagation of the small vortex in the $\varphi=$ const plane, as described above and shown in figure 1 . The two flow states depicted in figure 2 differ in their propagation velocity and propagation direction. The rotating wave at $\Gamma=1.10$ propagates retrogradely as indicated by the white arrow in figure $2(a)$. At larger $\Gamma$, the direction reverses and the wave propagates progradely. This behaviour is shown for $\Gamma=1.15$ in figure $2(b)$. The propagation velocity of the rotating wave depends continuously on the aspect ratio, and direction reversal occurs at a distinct $\Gamma$ with almost no change in the flow structure.

The process of direction reversal in an experimental system can be seen in the stability diagram (figure $3 a$ ) of a single-cell flow. In this diagram, the Reynolds number is held fixed at $R e_{i}=800$ and $\Gamma$ is plotted versus $\left|f_{\text {end }}\right|$, whereas the end plates rotate in the opposite direction as the inner cylinder. The fundamental frequency $f=m c /(2 \pi)$, with the wavenumber $m$ and the angular velocity $c$ of a rotating wave, reflects the dynamics in the laboratory frame. Note, that $c$ can be either positive or negative and this also holds for the fundamental frequency. Throughout this paper, frequencies are always scaled by the diffusion time $\tau=d^{2} / \nu$.

For $\Gamma \geqslant 1.125$, we observe that by increasing $\left|f_{\text {end }}\right|$, the steady single-cell flow undergoes a supercritical Hopf bifurcation at $\left|f_{\text {end }}\right| \approx 3$ (solid line in figure $3 a$ ) towards a rotating wave, such as the one displayed in figure $2(b)$. The positive frequency $(f>0)$ of the rotating wave above the Hopf bifurcation threshold in figure 3(a) indicates a 
(a)
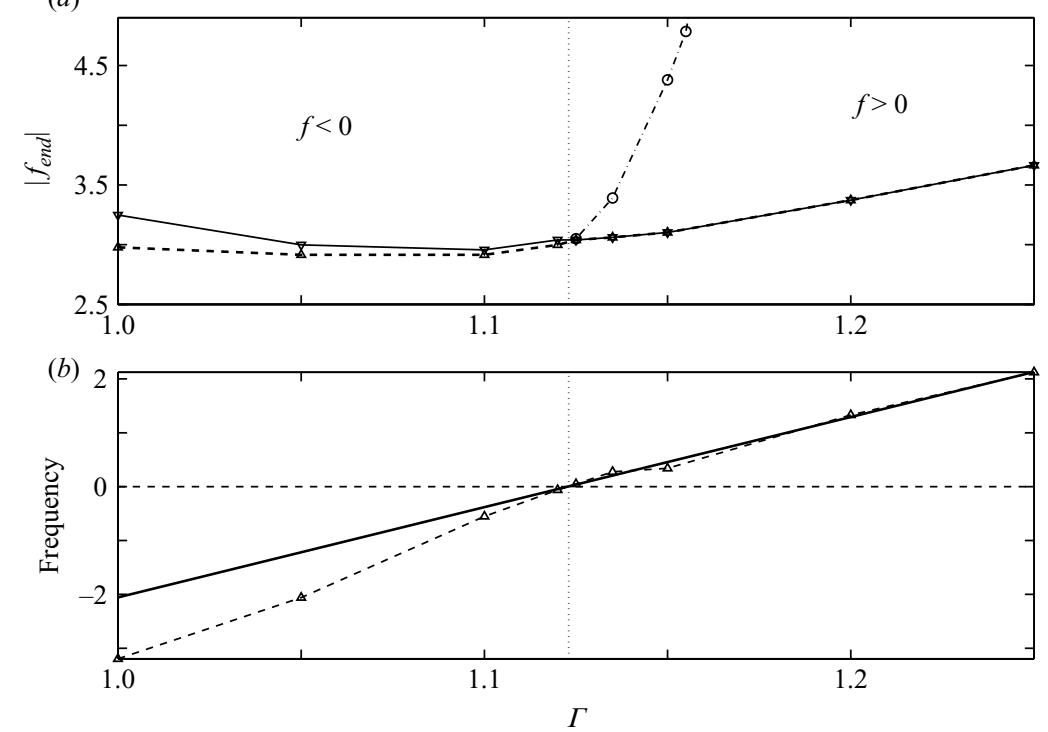

FiguRE 3. (a) Experimental stability diagram of single-cell flow at $R e_{i}=800$ : super- $(f>0)$ and subcritical $(f<0)$ Hopf bifurcation (solid curve) to rotating wave, disappearance of rotating wave from finite-amplitude (dashed curve), $f=0$ curve (dash-dotted curve), (b) oscillation frequency $(\triangle)$ of rotating wave at onset $(f>0)$ and decay from finite-amplitude $(f<0)$ (dashed curve), linear fit within the $f>0$ regime (solid curve).

prograde propagation. For $\Gamma \leqslant 1.12$, we found a retrograde propagation, such as the one displayed in figure $2(a)$ emerging out of the steady single-cell flow as $\left|f_{\text {end }}\right|$ is increased beyond a critical value (solid line in figure $3 a$ ). This is indicated by $f<0$. In this regime, a time-dependent flow occurs via a subcritical Hopf bifurcation. The rotating wave appears with finite-amplitude above the solid line and disappears from finite-amplitude below the dashed line at the critical $\left|f_{\text {end }}\right|$. The region of hysteresis becomes larger for smaller aspect ratios.

The frequency close to the Hopf bifurcation onset is shown in figure $3(b)$ as a function of the aspect ratio $\Gamma$. In the supercritical regime $(\Gamma \geqslant 1.125)$, the frequency is measured at the onset of the rotating wave whereas in the subcritical regime $(\Gamma \leqslant 1.12)$, the frequency of a finite-amplitude rotating wave is determined at the lowest border of hysteresis. We found an almost linear dependence in the supercritical regime (solid line). The value of aspect ratio where the frequency changes its sign from positive to negative is determined by a linear fit. Note that the negative frequencies in the subcritical regime refer to finite-amplitude rotating waves.

The dash-dotted line in figure 3(a) separates regions of different signs in the frequency, i.e. different propagation directions. This curve merges with the bifurcation curve within the interval $1.12<\Gamma<1.125$, i.e. between the experimentally determined borders of sub- and supercritical Hopf bifurcation regime.

In order to understand a symmetry-breaking bifurcation in a fluid flow, it is necessary to study the role of symmetry-breaking imperfections. Here, the rotational invariance, i.e. $S O(2)$, of the system is broken via a Hopf bifurcation. Small and unavoidable imperfections of the rotational invariance are always present in an experimental realization of Taylor-Couette flow and can have a strong effect on the dynamics of the flow. 

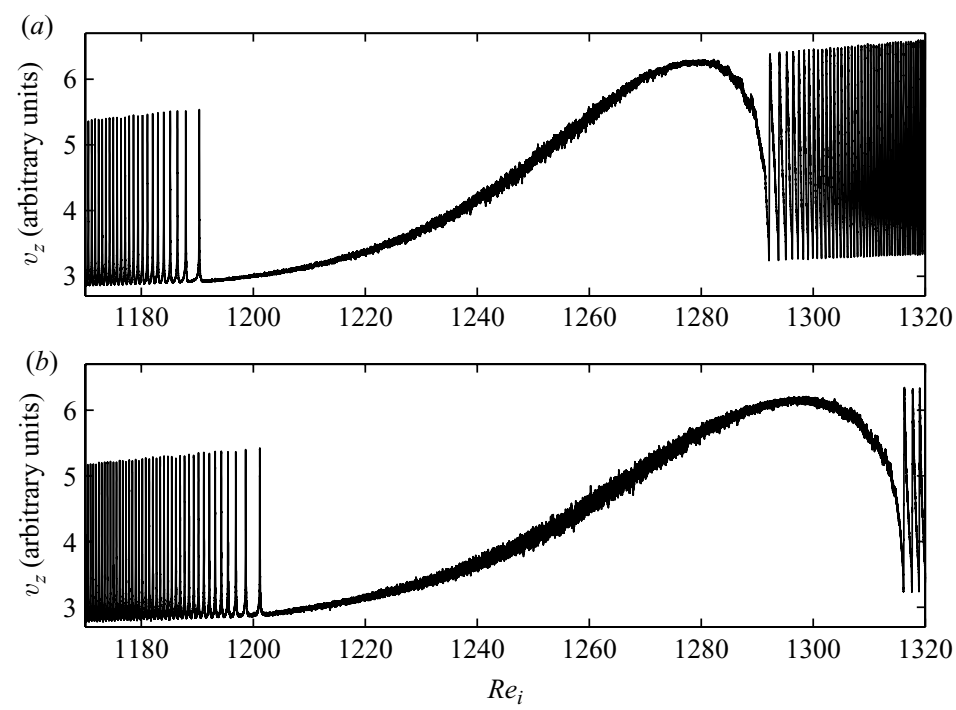

FIGURE 4. Reynolds number scan for $\Gamma=1.25$ and $f_{\text {end }}=0$ with $(a)$ a $0^{\circ}$ and $(b)$ a $0.08^{\circ}$ additional tilt of the upper end plate. A movie of a simulation of direction reversal of the rotating wave in the absence of azimuthal imperfections in available with the online version of the paper.

The time series depicted in figure 4 are recorded at $\Gamma=1.25$ and $f_{\text {end }}=0$ while the Reynolds number is increased quasi-statically from $R e_{i}=1170.0$ to $R e_{i}=1320.0$ within approx. $10 \mathrm{~h}$ (approx. 2400 diffusion times). This measurement technique of Reynolds-number scans allows us to distinguish between time-dependent and stationary regimes which appear in the scan as oscillations and noisy curves, respectively. It can be seen in figure 4(a) that within the interval $1190.0<R e_{i}<1290.0$, i.e. $\Delta R e_{i}=100$, the oscillation disappears and the flow becomes stationary.

We have also performed experiments with an additional (small) tilt of the upper end plate. A very small tilt angle of $0.08^{\circ}$ results, for example, in a shift of the interval of about $\Delta R e_{i}=15$ and an enlargement of about $\Delta R e_{i}=10$. Larger tilt angles are found to have a much stronger effect, but even very small imperfections of the rotational invariance produce a significant effect on the location and the size of the interval. Measurements of the zero frequency interval in different apparatuses with a comparable accuracy show significant differences in the width of this interval for the same aspect ratio. This provides further evidence for the sensitivity of the effect on small imperfections. Note that the additional (very small) breaking of the reflection symmetry is unlikely to be the cause for such a significant effect owing to the strong asymmetric nature of the single-cell flow.

In figure 4, two Reynolds-number scans for $\Gamma=1.25$ and $f_{\text {end }}=0$, showing the interval of stationary flow are depicted. A similar sequence is shown in figure 5(a) measured for $\Gamma=1.16$ also for the case of non-rotating end plates. The corresponding experimentally and numerically obtained oscillation frequencies are given in figure $5(c)$. The steady single-cell flow undergoes a supercritical Hopf bifurcation to a prograde rotating wave. This is indicated by the appearance of a broad black band in the scan which contains oscillations, such as those in figure 4 , but these oscillations are not resolved on this scale. Increasing $R e_{i}$ results in a decrease of the oscillation frequency which tends to zero at the lower border of the interval and 
(a)

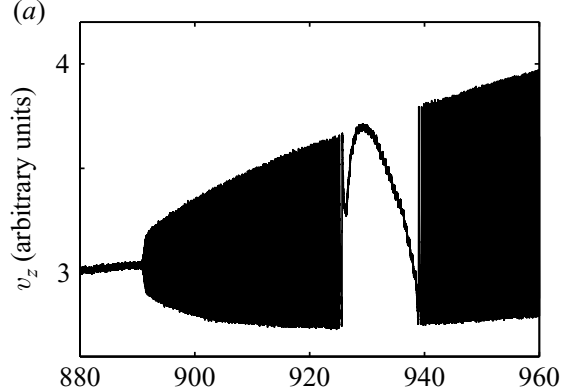

(c)

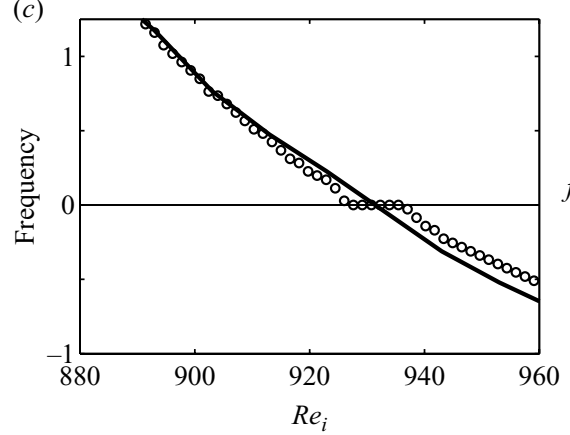

(b)

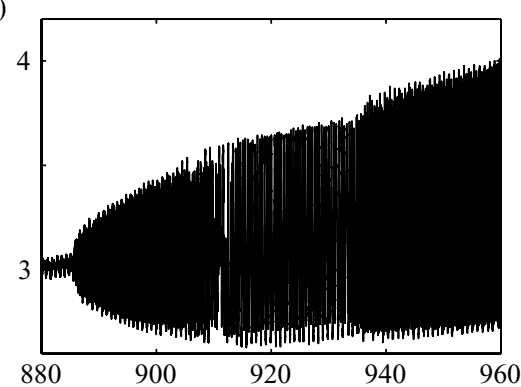

(d)

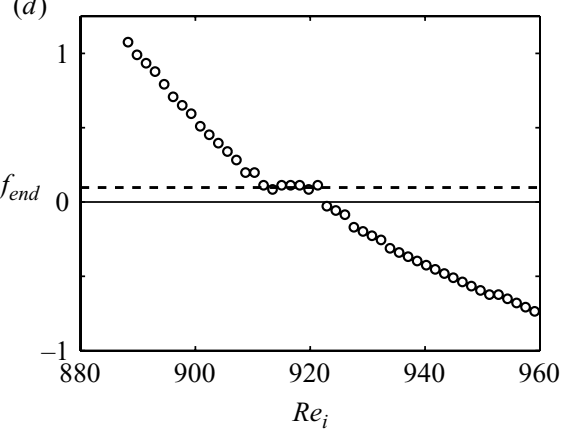

FIGURE 5. Reynolds-number scan at $\Gamma=1.16$ for $(a)$ stationary and $(b)$ slowly rotating end plates $\left(f_{\text {end }}=0.3\right)$. The corresponding oscillation frequencies of these experimental scans are plotted in $(c)$ and $(d)(\circ)$. The numerically determined values are additionally depicted as a solid line in $(c)$ and for the case of slowly rotating end plates, the rotation frequency of the end plates is indicated as a dashed line in $(d)$.

decreases further beyond the upper border towards more and more negative values. Within this interval, the frequency vanishes and the rotating wave is stationary. These experimental observations $(\circ)$ differ from the numerically obtained frequencies (solid line) where the frequency varies smoothly with the control parameter showing a distinct zero inside the experimentally determined band. Note that the numerically determined critical points of the rotating wave $R e_{i, c}$ are scaled to fit the difference (approx. $2 \%$ ) between the numerical and experimental onsets.

The results depicted in the figures 4 and $5(a, c)$ suggest that experimental imperfections are important in the case of non-rotating end plates which are absent in the numerical simulations. More evidence for the influence of these imperfections is provided by measurements with slowly rotating end plates. A Reynolds-number scan for the same aspect ratio but with very slowly rotating end plates $\left(f_{\text {end }}=0.1\right)$ is depicted in figure 5(b). A small shift in $R e_{i}$ for the onset of rotating waves can be seen and the $R e_{i}$-interval of zero frequency has disappeared. Furthermore, the black band within the scan appears more inhomogeneous than in figure 5(a) and the frequency of the end plates is clearly visible as a modulation of the scan, even below onset.

The corresponding frequencies are depicted in figure $5(d)$. A similar behaviour can be found in these experiments compared to figure $5(c)$ but with one qualitative difference: the plateau appears not at zero frequency, as for non-rotating end plates, but exactly at the frequency of the end plates (indicated by a horizontal dashed line in figure $5 d$ ). This observation leads to the conclusion that the rotating wave is coupled 

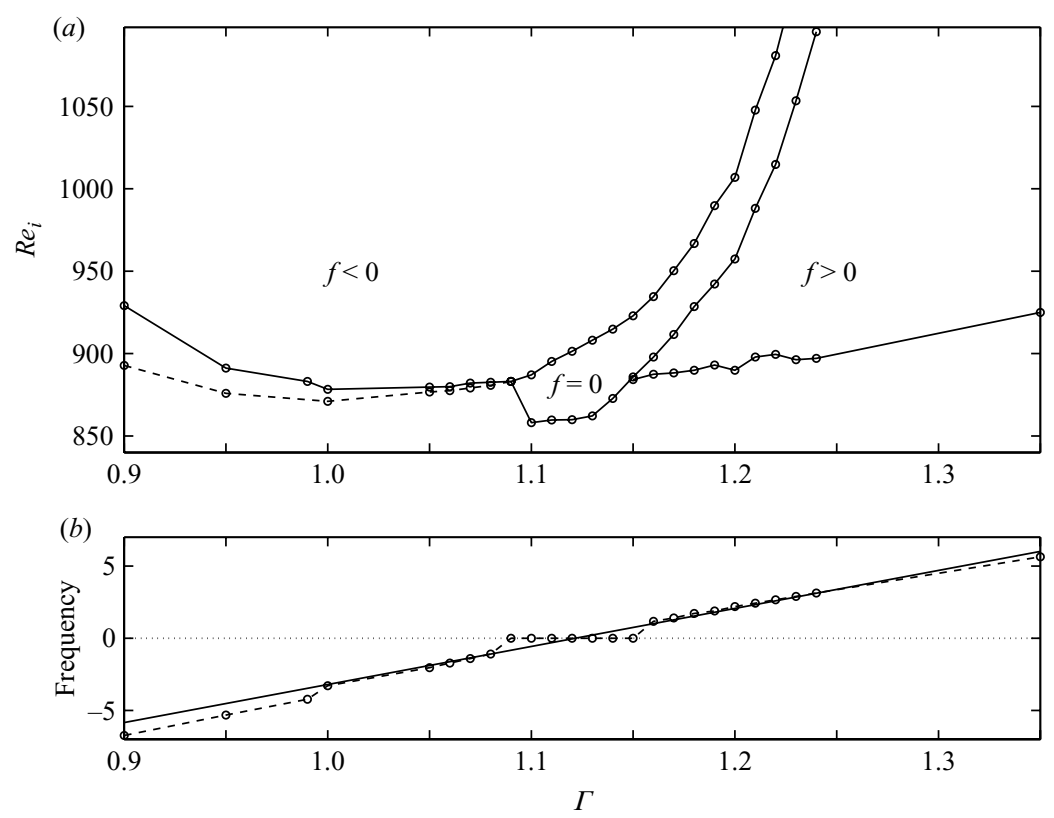

FIGURE 6. (a) Experimental stability diagram of the single-cell flow with stationary end plates. $(b)$ Oscillation frequencies of rotating wave $(\circ)$ at onset $(f \geqslant 0)$ and at lower stability border of the hysteresis $(f<0)$. Linear fit (solid line) close to the point of direction reversal. Dashed lines are added to guide the eyes.

to the imperfect end plates. Note that owing to the azimuthal wavenumber $m=2$, the rotation rates of the wave and the end plates differ by a factor of two.

The influence of imperfections on the bifurcation structure can be seen from the stability diagram of the one-cell flow with non-rotating end plates (figure $6 a$ ). For $\Gamma>1.15$, the steady single-cell flow undergoes a supercritical Hopf bifurcation at about $R e_{i} \approx 900$ (solid line) towards the prograde rotating wave $(f>0)$. For $\Gamma<1.09$, the retrograde propagating wave $(f<0)$ appears above a critical Reynolds number (solid line) but with hysteresis, i.e. from a subcritical Hopf bifurcation. This part of the bifurcation behaviour is qualitatively similar to that shown in figure 3(a); but here the prograde and the retrograde regimes are separated by a finite interval of zero frequency instead of a single curve in the $\left(R e_{i}, \Gamma\right)$-plane. The results shown in figure 5 suggest that the rotating wave does not disappear within this band, but remains stationary in the laboratory frame. In the vicinity of direction reversal $(1.09 \leqslant \Gamma \leqslant 1.15)$, the rotating wave is found to appear supercritically in the flow as indicated by the lower line bounding the $f=0$-interval in figure $6(a)$. This behaviour can also be seen from the dependence of frequency at the onset (or decay from finite-amplitude) on the aspect ratio in figure $6(b)$.

\section{Conclusions}

We found that a wave can change its direction of rotating from prograde to retrograde in the inertial frame. This is in contrast to other types of rotating waves in Taylor-Couette flow, such as the classical wavy vortex flow, which always propagate progradely in the inertial frame. The frequency of this rotating wave is found to depend continuously on the control parameters $\left(R e, \Gamma, f_{\text {end }}\right)$ and the reversal of 
direction occurs at a distinct point (if only one parameter is varied and the other two are kept fixed) without almost any change in the flow structure. This behaviour is studied experimentally and confirmed by numerical three-dimensional Navier-Stokes simulations. At onset, the point of direction reversal is accompanied with a change of the Hopf bifurcation from super- to subcritical. Such changes have been referred to as a generalized Hopf bifurcation (Guckenheimer \& Holmes 1983) or a tricritical phenomena (Aitta, Ahlers \& Cannell 1985).

We found that small imperfections of the rotational invariance have a crucial influence on this bifurcation. Phase locking between the rotating wave and imperfect rotating and non-rotating end plates within a finite interval in the control parameter space can occur for comparable frequencies. Since symmetry-breaking imperfections are an integral part of the description of a bifurcation event, a third control parameter is required in order to unfold the bifurcation structure underlying direction reversal.

Though in principle, a rotating wave can always be considered as steady flow in a certain co-rotating frame, the reversal of direction in the inertial (laboratory) frame is organized in a bifurcation of higher codimension.

This provides an explanation for the zero frequency interval within a rotating wave regime in small-aspect-ratio Taylor-Couette flow. Our results therefore highlight the crucial role of symmetry-breaking imperfections as an integral part of a complete description of bifurcation in fluid dynamics.

We acknowledge support from the Deutsche Forschungsgemeinschaft.

\section{REFERENCES}

Abshagen, J., Heise, M., Langenberg, J. \& Pfister, G. 2007 Imperfect Hopf bifurcation in spiral Poiseuille flow. Phys. Rev. E 75(1), 016309.

Ahlers, G., Cannell, D. S. \& Dominguez Lerma, M. A. 1983 Possible mechanism for the transition to wavy Taylor-vortex flow. Phys. Rev. A 27, 1225-1227.

Aitta, A., Ahlers, G. \& Cannell, D. S. 1985 Tricritical phenomena in rotating Couette-Taylor flow. Phys. Rev. Lett. 54, 673-676.

Andereck, C. D., Lui, S. S. \& Swinney, H. L. 1986 Flow regimes in a circular Couette system with independently rotating cylinders. J. Fluid Mech. 164, 155-183.

Coles, D. 1965 Transition in circular Couette flow. J. Fluid Mech. 21, 385-425.

Crawford, J. D. \& KNOBloch, E. 1988 On degenerate Hopf bifurcation with broken O(2)-symmetry. Nonlinearity 1, 617-652.

DiPrima, R. C. \& Swinney, H. L. 1981 Instabilities and transition in flow between concentric cylinders. In Hydrodynamic Instabilities and the Transition to Turbulence (ed. H. L. Swinney \& J. P. Gollub), pp. 139-180. Springer.

Edwards, W. S., BeAne, S. R. \& Varme, S. 1991 Onset of wavy vortices in the finite-length Couette-Taylor problem. Phys. Fluids A 3, 1510-1518.

Egbers, C. \& Pfister, G. 2000 Physics of Rotating Fluids. Springer.

Gerdts, U., von Stamm, J., Buzug, Th. \& Pfister, G. 1994 Axisymmetric time-dependent flow in the Taylor-Couette system. Phys. Rev. E 49, 4019-4026.

Gill, A. E. 1982 Atmosphere-Ocean Dynamics. Academic.

van Gils, S. \& Mallet-Paret, J. 1986 Hopf bifurcation with symmetry: travelling and standing waves on the circle. Proc. R. Soc. Edin. A 104, 279-307.

Goldstein, H. F., Knobloch, E., Mercader, I. \& Net, M. 1993 Convection in a rotating cylinder. Part 1. Linear theory for moderate Prandtl numbers. J. Fluid Mech. 248, 583-604.

Golubitsky, M., Stewart, I. \& Schaeffer, D. G. 1988 Singularities and Groups in Bifurcation Theory, vol 2. Springer.

Guckenheimer, J. \& Holmes, P. 1983 Nonlinear Oscillations, Dynamical Systems, and Bifurcations of Vector Fields. Springer. 
Hoffmann, Ch., LÜCKe, M. \& Pinter, A. 2005 Spiral vortices traveling between two rotating defects in the Taylor-Couette system. Phys. Rev. E 72 (5), 056311.

Iooss, G. 1985 Secondary bifurcations of Taylor vortices into wavy inflow and wavy outflow boundaries. J. Fluid Mech. 173, 273-288.

Jones, C. A. 1981 Nonlinear Taylor vortices and their stability. J. Fluid Mech. 102, 249-261.

Jones, C. A. 1985 The transition to wavy Taylor vortices. J. Fluid Mech. 157, 135-162.

KNOBLOCH, E. 1996 Symmetry and instability in rotating hydrodynamic and magnetohydrodynamic flows. Phys. Fluids 8, 1446-1454.

Marcus, P. S. 1984 Simulation of Taylor-Couette flow. Part 2. Numerical results for wavy-vortex flow with one travelling wave. J. Fluid Mech. 146, 65-113.

Marcus, P. S. \& Tuckerman, L. S. 1987 Simulation of flow between concentric rotating spheres. Part 2. Transitions. J. Fluid Mech. 185, 31-65.

Marques, F. \& Lopez, J. M. 2006 Onset of three-dimensional unsteady states in small-aspect-ratio Taylor-Couette flow. J. Fluid Mech. 561, 255-277.

Marques, F., Lopez, J. M. \& Shen, J. 2002 Mode interaction in an enclosed swirling flow: a double Hopf bifurcation between azimuthal wavenumber 0 and 2. J. Fluid Mech. 455, 263-281.

Marques, F., Mercader, I., Batiste, O. \& Lopez, J. M. 2007 Centrifugal effects in rotating convection: axisymmetric states and three-dimensional instabilities. J. Fluid Mech. 580, 303318.

Moisy, F., Doare, O., Pasutto, T., Daube, O. \& Rabaud, M. 2004 Experimental and numerical study of the shear layer instability between two counter-rotating discs. J. Fluid Mech. 507, $175-202$.

Mullin, T. 1985 Onset of time-dependence in Taylor-Couette flow. Phys. Rev. A 31, 1216.

Mullin, T. 1994 The Nature of Chaos. Oxford University Press.

Mullin, T. \& Benjamin, T. B. 1980 Transition to oscillatory motion in the Taylor experiment. Nature 288, 567.

NagATA, M. 1988 On wavy instabilities of the Taylor-vortex flow between corotating cylinders. J. Fluid Mech. 188, 585-598.

Nore, C., Tuckerman, L. S., Daube, O. \& Xin, S. 2003 The 1:2 mode interaction in exactly counter-rotating von Kármán swirling flow. J. Fluid Mech. 477, 51-88.

Pfister, G., Schulz, A. \& Lensch, B. 1991 Bifurcation and a route to chaos of a one-vortex-state in the Taylor-Couette flow. Eur. J. Mech. B/Fluids 10 (2), 247-252.

Serre, E., Crespo del Arco, E. \& Bontoux, P. 2001 Annular and spiral patterns in flows between rotating and stationary disks. J. Fluid Mech. 434, 65-100.

Swinney, H. L. \& Gollub, J. P. 1981 Hydrodynamic Instabilities and the Transition to Turbulence. Springer.

Zhong, F., Ecke, R. E \& Steinberg, V. 1991 Asymmetric modes and the transition to vortex structures in rotating Rayleigh-Bénard convection. Phys. Rev. Lett. 67, 2473-2476. 